\title{
Mudanças Climáticas e a Biodiversidade dos Biomas Brasileiros: Passado, Presente e Futuro
}

\author{
Climate Change and Biodiversity of Brazilian Biomes: Past, Present, and Future
}

\author{
Alexandre Aleixo ${ }^{1 *}$, Ana Luisa Albernaz ${ }^{2}$, Carlos Eduardo Viveiros Grelle ${ }^{3}$, \\ Mariana Moncassim Vale $^{3} \&$ Thiago Fernando Rangel ${ }^{4}$
}

\author{
${ }^{1}$ Coordenação de Zoologia, Museu Paraense Emílio Goeldi, CP 399, CEP 66040-170, Belém, PA, Brasil \\ ${ }^{2}$ Coordenação de Ciências da Terra e Ecologia, Museu Paraense Emílio Goeldi, \\ CP 399, CEP 66040-170, Belém, PA, Brasil \\ ${ }^{3}$ Departamento de Ecologia, Universidade Federal do Rio de Janeiro - UFRJ, \\ CP 68020, CEP 21941-902, Rio de Janeiro, RJ, Brasil \\ ${ }^{4}$ Departamento de Ecologia, Instituto de Ciências Biológicas, Universidade Federal de Goiás - UFG, \\ CP 131, CEP 74001-970, Goiânia, GO, Brasil
}

\begin{abstract}
Os biomas brasileiros abrigam uma porção significativa da biodiversidade mundial, constituindo importantes centros de biodiversidade pela combinação de altos níveis de riqueza e endemismo. No entanto, essa rica biodiversidade vem sendo crescentemente ameaçada por atividades antrópicas, principalmente aquelas ligadas à conversão das paisagens naturais em áreas de produção agro-pecuária e ocupação imobiliária. Altíssimos níveis de devastação ambiental já colocaram dois biomas brasileiros - a Mata Atlântica e o Cerrado - na lista dos "Hotspots" de biodiversidade, que são conjuntos de ecorregiões prioritárias para conservação em nível mundial (Myers et al. 2000). Para piorar a situação, o conhecimento sobre a real diversidade dos grupos biológicos que compõe a biodiversidade brasileira ainda pode ser considerado bastante incipiente, mesmo para aqueles grupos que tradicionalmente sempre foram considerados bem conhecidos, como é o caso das aves (Vale et al. 2008), o que pode inviabilizar seu uso como fonte confiável de informações para planejamentos sistemáticos e desenvolvimento de políticas de conservação (Aleixo 2010).
\end{abstract}

Além das alterações recentes nas paisagens naturais, mudanças climáticas em curso e previstas constituem um segundo fator de ameaça à biodiversidade dos biomas brasileiros, com especial ênfase para aqueles predominantemente florestais e com maior riqueza de espécies e endemismo: a Amazônia e a Mata Atlântica. Apesar da sua importância, a produção científica nesta área do conhecimento é ainda pequena se comparada

${ }^{\star}$ Send correspondence to: Alexandre Aleixo Coordenação de Zoologia, Museu Paraense Emílio Goeldi, CP 399, CEP 66040-170, Belém-PA, Brasil E-mail: aleixo@museu-goeldi.br à outras relacionadas com a Biologia da Conservação (Grelle et al. 2009). Na América do Sul, temperaturas mais altas e uma maior duração da estação seca poderão aumentar a frequência de estiagens sazonais rigorosas iniciadas pelo episódio El Niño / Oscilação Sul (ENSO) e de anomalias da temperatura da superfície do mar (SST) no Atlântico, contribuindo para incêndios cada vez mais frequentes e intensos, os quais ameaçarão a distribuição e integridade ambiental dos biomas brasileiros, em particular os predominantemente florestais (Marengo et al. 2009).

Um conhecimento essencial para o desenvolvimento de políticas de minimização dos efeitos das mudanças climáticas sobre a biodiversidade brasileira é a reconstrução do grau de suscetibilidade e resposta das biotas dos diferentes biomas às alterações climáticas intensas de passado recente, como aquelas ocorridas ao longo dos últimos 2 milhões de anos (i.e. período Quaternário). Dados recentes sobre a filogeografia e modelagem de nicho ecológico obtidos para várias linhagens de organismos da Amazônia e Mata Atlântica, dão uma ideia da suscetibilidade dos diferentes biomas Enquanto para alguns grupos os últimos ciclos de diversificação correlacionaram-se aparentemente com essas alterações climáticas recentes, em outros, estas pouco ou nada contribuíram para seu padrão contemporâneo de riqueza de espécies e distribuição geográfica (Carnaval et al. 2009, Antonelli et al. 2010). O entendimento das variáveis determinantes deste padrão dicotômico de resposta frente às alterações climáticas intensas, em curtos períodos de tempo, adquire uma importância fundamental no contexto do aquecimento global já verificado e previsto para várias regiões do planeta, inclusive para os biomas brasileiros (Marengo et al. 2009). 


\section{Mudanças Climáticas e Biodiversidade: o Passado como Chave para Entender o Futuro}

Uma das hipóteses até hoje mais difundidas e intensamente debatidas sobre a origem da grande biodiversidade Neotropical e brasileira contemporâneas (i.e. hipótese dos refúgios pleistocênicos), coloca as mudanças climáticas no centro do processo de geração de barreiras que promovem a vicariância e geração de novas linhagens e espécies, num processo conhecido como cladogênese (Haffer 2008). Segundo a hipótese, as máximas glaciais, como aquela ocorrida há cerca de 20 mil anos atrás, teriam levado a temperaturas mais baixas e a uma drástica redução na pluviosidade média de alguns biomas brasileiros, com inferida redução e fragmentação dos biomas florestais e expansão de formações abertas; já os períodos interglaciais, com características semelhantes ao clima atual, teriam fornecido, de modo inverso, condições ideais para a expansão dos biomas florestais e a retração de formações abertas (Vonhof \& Kaandorp 2010). O efeito destes ciclos nas populações dos diferentes organismos teria variado, dependendo do bioma. Durante períodos glaciais, populações de espécies florestais teriam sua distribuição fragmentada, ficando isoladas em "refúgios" florestais e se diferenciando uma das outras, ao passo que espécies de ambientes abertos expandiriam suas distribuições acompanhando a expansão destes ambientes. Em períodos inter-glaciais, o oposto teria ocorrido e espécies florestais recém diferenciadas expandiriam suas distribuições, entrando em contato, enquanto que espécies de área abertas teriam suas distribuições fragmentadas e passariam a se diferenciar pelo isolamento.

Embora a hipótese dos refúgios tenha sido aquela mais discutida para a biota Neotropical até hoje e inclusive corroborada por diferentes estudos (ver revisões em Haffer (2008) e Antonelli et al. (2010)), diferentes estudos paleoclimáticos e filogenéticos falsificaram algumas das suas mais importantes previsões, evidenciando claramente as limitações desta hipótese. Por exemplo, alguns estudos palinológicos não encontraram evidências de vegetações abertas durante a Última Máxima Glacial (UMG; 20 mil anos atrás) em grandes extensões da Amazônia ocidental, conforme previsto pela hipótese dos refúgios, mas sim indícios de uma cobertura florestal similar à atual (Colinvaux 1993). De modo análogo, a maior parte das datações obtidas para a separação de espécies irmãs de vertebrados no bioma Amazônia, aponta para uma origem bem anterior à UMG, demonstrando que períodos glaciais, por si só, não seriam responsáveis pelo mais recente ciclo de cladogênese nestas linhagens (Antonelli et al. 2010).

Independentemente de ter contribuído decisivamente ou não para formação de novas linhagens e espécies em diferentes contextos, é indiscutível que as mudanças climáticas ocorridas durante a UMG afetaram de modo bastante intenso o nicho ecológico potencial ocupado por espécies associadas aos biomas brasileiros. Isso pode ter ocasionado, no mínimo, grandes mudanças na distribuição geográfica destas espécies em relação ao contexto atual. Estima-se que, particularmente a Amazônia e Mata Atlântica, ficaram bastante reduzidas em relação à sua distribuição atual, conforme revelado pela distribuição do nicho ecológico potencial de várias linhagens associadas a esses biomas durante períodos glaciais, incluindo a UMG (e.g. Carnaval et al. 2009). Talvez um dos pontos mais obscuros sobre os efeitos dos ciclos glaciais de mudanças climáticas na biodiversidade dos biomas brasileiros seja o seu potencial de promover extirpações locais e até extinções completas de linhagens e espécies. Infelizmente, as assinaturas desses eventos não podem ser recuperadas de modo direto através de reconstruções filogenéticas (Rabosky 2010). De todo modo, mesmo com a drástica redução da cobertura dos biomas Amazônia e Mata Atlântica na UMG, isso parece não ter sido suficiente para a extinção de várias linhagens modernas, conforme evidenciado por diversos estudos filogenéticos e de modelagem do nicho ecológico pretérito e contemporâneo.

Desse modo, três grandes questões inter-relacionadas se impõem hoje no estudo da biodiversidade brasileira num contexto de mudanças climáticas globais intensas em curso e previstas para o futuro próximo: (1) quais as características ecológicas das linhagens que apresentam uma resistência natural ou vulnerabilidade à mudanças climáticas, conforme revelado por seu passado evolutivo?, (2) qual o limiar de alteração climática necessário para gerar não apenas alterações em padrões de distribuição, mas também alterações nas fitofisionomias e extinções de linhagens inteiras nos biomas brasileiros? e (3) mudanças climáticas podem continuar atuando, como no passado, como fatores que contribuem, em diferentes contextos, para a geração de novas linhagens e espécies em biomas cada vez mais descaracterizados por paisagens antropizadas?

\section{O Componente "Biodiversidade" da Rede Brasileira de Pesquisas sobre Mudanças Climáticas Globais: Modelando o Presente para Diagnosticar o Futuro}

A Rede Brasileira de Pesquisas sobre Mudanças Climáticas Globais (conhecida como Rede-Clima) foi criada em dezembro de 2007 e tem sua sede no Instituto Nacional de Pesquisas Espaciais (INPE) em São José dos Campos (SP), contando com diversas atribuições na formulação e acompanhamento de políticas públicas sobre Mudanças Climáticas Globais no Brasil, inclusive "estudar alternativas de adaptação dos sistemas sociais, econômicos e naturais do Brasil às mudanças climáticas”.

Para esse fim, foi constituída uma sub-rede dentro da Rede-Clima, com foco na biodiversidade dos biomas brasileiros e cuja finalidade principal é entender melhor os efeitos das mudanças climáticas sobre a biota brasileira, 
possibilitando a elaboração de estratégias para minimizar seus efeitos deletérios. Atualmente, a sub-rede Biodiversidade da Rede Clima executa projetos de pesquisa na sua temática de especialidade nos biomas Amazônia, Cerrado e Mata Atlântica, devendo em breve expandir sua rede de colaboradores para os demais biomas brasileiros. Neste primeiro momento, os projetos em execução na sub-rede têm três focos principais: (1) usando modelagem de nicho ecológico, mapear possíveis redistribuições geográficas para determinadas linhagens de organismos, sob cenários diversos de emissão de gases de efeito estufa, identificando espécies e áreas prioritárias para a conservação num futuro marcado por mudanças climáticas em andamento; (2) reconstruir os contextos temporal, espacial e ecológico do histórico de diversificação de determinadas linhagens de organismos, com vistas a identificar um conjunto de variáveis bio-geo-climáticas responsáveis pela diversificação e não extinção destes grupos no passado, que podem ser determinantes para a sua sobrevivência num futuro marcado pelas mudanças climáticas em andamento e (3) modelar o comportamento de diferentes tipos de fitofisionomias dos biomas brasileiros frente à mudanças climáticas, com o objetivo principal de prever alterações na sua distribuição futura.

Para atingir estes objetivos, é necessária uma abordagem que integre diversas disciplinas, como, por exemplo, a sistemática filogenética molecular e a modelagem de nicho ecológico Grinneliano. Estudos das relações filogenéticas entre táxons próximos, muitos deles considerados atualmente como subespécies ou complexos de espécies, são importantes para se delimitar apropriadamente espécies e evidenciar padrões temporais e espaciais de diversificação. A partir desses resultados, é possível verificar se há padrões comuns que possam ser relacionados a eventos históricos. Para compreender os processos históricos mais recentes, em especial os relacionados a alterações climáticas Pleistocênicas, são necessários estudos populacionais, intra-específicos, que podem indicar padrões demográficos comuns a diferentes grupos que ocorrem nas mesmas regiões geográficas, além de técnicas de modelagem de nicho ecológico com base na distribuição geográfica das linhagens investigadas.

A sub-rede biodiversidade da Rede Clima espera gerar um conjunto de dados acadêmicos/científicos com resolução e robustez inéditos sobre a origem, evolução, ecologia histórica e redistribuição em cenários de mudanças climáticas para linhagens de diversos organismos dos biomas brasileiros, que subsidiem diretamente a elaboração de estratégias de conservação futuras eficientes para estes biomas num cenário crescente de mudanças climáticas. Neste sentido, a sub-rede biodiversidade pretende aglutinar um número cada vez maior de pesquisadores e instituições focados em seus objetivos, além de promover encontros entre especialistas com o objetivo de promover a troca de experiências, o compartilhamento de dados e a formulação de sínteses de aproveitamento imediato para formulação de políticas públicas na área da conservação do meio-ambiente e biodiversidade.

\section{Agradecimentos}

Os autores são gratos ao Ministério da Ciência e Tecnologia (MCT) e Instituto Nacional de Pesquisas Espaciais (INPE) pelo apoio através da Rede-Clima. A. Aleixo e A. L. Albernaz agradecem ao CNPq pelo apoio através do "Instituto Nacional de Ciência e Tecnologia (INCT) em Biodiversidade e Uso da Terra da Amazônia" (auxílio nº. 574008/2008-0). A. Aleixo recebe uma bolsa de produtividade em pesquisa do $\mathrm{CNPq}$ (auxílio n ${ }^{\circ}$. 310593/2009-3). C. E. V Grelle agradece ao CNPq pelos auxílios e bolsa de produtividade e a FAPERJ pelo auxílio Jovem Cientista do Estado do Rio de Janeiro. M. M. Vale agradece ao CNPq pela bolsa de Pós-doutorado Júnior.

\section{Referências}

Aleixo A, 2010. "Incerteza taxonômica" na biodiversidade amazônica: por que resolvê-la é imprescindível para a conservação do bioma? In: Themoteo R (Ed.). Cadernos Adenauer - Amazônia e desenvolvimento sustentável. 4 ed. Rio de Janeiro: Fundação Konrad Adenauer. v. 10, p. 35-57.

Antonelli A et al., 2010. Molecular studies and phylogeography of Amazonian tetrapods and their relation to geological and climatic models. In Hoorn C \& Wesselingh F (Ed.). Amazonia, Landscape and Species Evolution: a look into the past. Blackwell Publishing. p. 386-404.

Carnaval AC et al., 2009. Stability predicts genetic diversity in Brazilian Atlantic forest hotspot. Science, 323:785-789.

Colinvaux PA, 1993. Pleistocene biogeography and diversity in tropical forests of South America. In: GoldBlatt, P (Ed.). Biological relationships between Africa and South America. New Haven: Yale University Press. p. 473-499.

Grelle CEV et al., 2009. Uma década de Biologia da Conservação no Brasil. Oecologia Brasiliensis, 13:420-433.

Haffer J, 2008. Hypotheses to explain the origin of species in Amazonia. Brazilian Journal of Biology, 68:917-947.

Marengo JA et al., 2009. Global warming and climate change in Amazonia. In: Keller M et al., (Ed.) Amazonia and Global Change. Washington DC: American Geophysical Union. v. 186, p. $262-273$.

Myers $\mathrm{N}$ et al., 2000. Biodiversity hotspots for conservation priorities. Nature, 403:853-858.

Rabosky DL, 2010. Extinction rates should not be estimated from molecular phylogenies. Evolution, 64:1816-1824.

Vale MM et al., 2008. Effects of Future Infrastructure development on threat status and occurrence of amazonian birds. Conservation Biology, 22:1006-1015.

Vonhof HB \& Kaandorp RJG, 2010. Climate variation in Amazonia during the Neogene and the Quaternary. In: Hoorn C and Wesselingh F (Ed.). Amazonia, Landscape and Species Evolution: a look into the past. Blackwell Publishing. p. 201-210.

Recebido: Novembro 2010

Primeira Decisão: Novembro 2010 Aceito: Novembro 2010 